\title{
A platinum black-modified microelectrode for in situ olanzapine detection in microliter volumes of undiluted serum
}

\author{
Rajendra P. Shukla ${ }^{1} \cdot$ Robert H. Belmaker ${ }^{2} \cdot$ Yuly Bersudsky $^{2} \cdot$ Hadar Ben-Yoav $^{1}$ (i)
}

Received: 10 October 2019 / Accepted: 28 December 2019 / Published online: 6 January 2020

(c) The Author(s) 2020

\begin{abstract}
Olanzapine is a thienobenzodiazepine compound. It is one of the newer types of antipsychotic drugs used in the treatment of schizophrenia and other psychotic disorders. Several methods have been reported for analyzing olanzapine in its pure form or combined with other drugs and in biological fluids. These methods include high-performance liquid chromatography and liquid chromatography-tandem mass spectroscopy. Although many of the reported methods are accurate and sensitive, they require the use of sophisticated equipment, lack in situ analysis, and require expensive reagents. Moreover, several of these methods are cumbersome, require prolonged sample pretreatment, strict control of $\mathrm{pH}$, and long reaction times. Here we present the development of a miniaturized electrochemical sensor that will enable minimally invasive, real-time, and in situ monitoring of olanzapine levels in microliter volumes of serum samples. For this purpose, we modified a microfabricated microelectrode with a platinum black film to increase the electrocatalytic activity of the microelectrode towards olanzapine oxidation; this improved the overall selectivity and sensitivity of the sensor. We observed in recorded voltammograms the anodic current dose response characteristics in microliter volumes of olanzapine-spiked serum samples that resulted in a limit of detection of $28.6 \pm 1.3 \mathrm{nM}$ and a sensitivity of $0.14 \pm 0.02 \mu \mathrm{A} / \mathrm{cm}^{2} \mathrm{nM}$. Importantly, the platinum black-modified microelectrode exhibited a limit of detection that is below the clinical threshold (65-130 nM). Further miniaturizing and integrating such sensors into point-of-care devices provide real-time monitoring of olanzapine blood levels; this will enable treatment teams to receive feedback and administer adjustable olanzapine therapy.
\end{abstract}

Keywords Antipsychotic olanzapine $\cdot$ Serum $\cdot$ Electrochemical sensors $\cdot$ Point-of-care testing $\cdot$ Therapeutic drug monitoring $\cdot$ Reduced graphene oxide

\section{Introduction}

Olanzapine (OLZ) is the most commonly used second-generation antipsychotic medication approved by the FDA for Schizophrenia treatment. Patients with mental health problems are advised to take this medicine once a day; the doses range from 5 to $10 \mathrm{mg}$, depending on the patient's illness condition (Lin et al. 2017). Therapeutic drug monitoring of this drug is very important in order to improve the efficacy

Hadar Ben-Yoav

benyoav@bgu.ac.il

1 Nanobioelectronics Laboratory (NBEL), Department of Biomedical Engineering and Ilse Katz Institute of Nanoscale Science and Technology, Ben-Gurion University of the Negev, 8410501 Beer-Sheva, Israel

2 Department of Psychiatry, Mental Health Center, Ben-Gurion University of the Negev, 8410501 Beer-Sheva, Israel of the treatment ( $\mathrm{Lu}$ et al. 2018). Current state-of-the art technology, which is used to monitor Olanzapine levels in patients, includes high-performance liquid chromatography and liquid chromatography-tandem mass spectroscopy. Unfortunately, these methods require frequent and invasive blood draws from patients. This is a painful process that requires trained personnel to deal with sophisticated instruments, thus increasing the cost of treatment. In addition, it is a time-consuming process that includes admitting patients and obtaining a report from the central labs (Ni et al. 2018; Wu et al. 2019). Overall, it is an added burden on the patients in terms of time and money.

Olanzapine is a redox active molecule (it can donate and receive electrons); therefore, electrochemical sensors can provide an alternate solution to the current state-of-theart analytical methods due to their simple design and easy manufacturing process as well as their high sensitivity and cost effectiveness, compared with the currently available 
analytical tools for analyzing redox molecules (Merli et al. 2012). Electrochemical sensors have been previously used to detect OLZ; however, they suffer from several challenges. Currently no available sensor can monitor the OLZ blood levels in situ and at the point of care. El-Shal reported the electrochemical sensing of OLZ using a glassy carbon electrode in Britton-Robinson buffer ( $\mathrm{pH}$ 2.0) solution; the limit of detection (LOD) was $1 \times 10^{-8} \mathrm{M}$ (El-Shal 2013). However, El-Shal's research did not detect OLZ in biological samples, such as serum. Arvand and Palizkar reported on the electrochemical detection of OLZ using an amine-functionalized $\mathrm{TiO}_{2} /$ multi-walled carbon nanotube consisting of a composite-modified glassy carbon platform (Arvand and Palizkar 2013). They reported a LOD value of $90 \mathrm{nM}$ in phosphate buffered saline $(0.1 \mathrm{M}, \mathrm{pH} 5)$ and applied the same platform to recover OLZ in diluted serum samples in same buffer solution. The effect of solution $\mathrm{pH}$ on OLZ oxidation was studied; it was found that the solution $\mathrm{pH}$ significantly influences the oxidation peak current. The oxidation peak current was maximum at $\mathrm{pH} 5.0$; however, it decreased at higher $\mathrm{pH}$ values due to proton deficiency. As a result, pH 5.0 was selected for this study. Ahmed et al. demonstrated OLZ detection with a modified carbon paste sensor electrode incorporating gold nanoparticles and glutamine in a micellar medium (Ahmed et al. 2015). They reported an LOD value of $3.58 \times 10^{-9} \mathrm{M}$ in Britton-Robinson buffer $(\mathrm{pH}$ 7.0) and demonstrated the recovery of OLZ in urine samples. However, this study did not show how interfering species affect the detection of OLZ in biological samples within their therapeutic range. For a point-of-care device, a smaller sample volume (a few microliters) is also required. Arvand et al. reported an electrochemical sensor based on $\mathrm{Fe}_{3} \mathrm{O}_{4} @ \mathrm{Ag}$ core/shell magnetic nanoparticles-a modified carbon paste electrode that was used to investigate the electrochemical behavior of olanzapine in acetate buffer $(\mathrm{pH}$ 4.3) (Arvand et al. 2015). They reported an LOD value of $0.0018 \mu \mathrm{M}$ in acetate buffer and used the same sensor to recover OLZ from tablets and in diluted serum samples from schizophrenia patients. However, this study also did not demonstrate real-time and in situ analysis of OLZ in microliter volume samples at the point of care. Recently, the group of Rouhani et al. demonstrated OLZ detection in diluted serum samples using a carbon paste electrode. (Rouhani and Soleymanpour 2019) Although the results of these studies are promising, they did not demonstrate an improved sensing performance in micro-systems integrated with microelectrodes using micro-liter sample volumes. Several challenges are associated with microscale analytical devices, including low signal-to-background ratios as well as physical and chemical effects (e.g., capillary forces, surface roughness, and chemical interactions of the construction material with the reaction processes). In addition, the sensitivity and specificity of these micro-systems are reduced when dealing with biological fluids (e.g., urine and serum) due to the presence of other electro-active species in the solution. These challenges of using an analytical device limit the ability to detect low levels of drugs in biological fluids. However, by utilizing a miniaturized lab-on-a-chip electrochemical micro-system, schizophrenia treatment outcomes can be improved.

Here we report on a platinum black (PB) -modified microelectrode for the in situ detection of OLZ in microliter volumes of undiluted serum samples (Fig. 1). Platinum black film has been used for electrochemical sensing applications because of its high conductivity and electrocatalytic activity (Li et al. 2013; Seo et al. 2008). The platinum blackmodified microelectrode enhances the effective surface area, resulting in significantly increased OLZ oxidation current. The increased oxidation process of OLZ results in better sensitivity and improved LOD values in the buffer as well as in undiluted serum samples. Interestingly, a bare $\mathrm{Au}$ microelectrode was unable to differentiate between different OLZ concentrations, whereas a PB-modified microelectrode exhibited a positive dose response with a high background. More specifically, the PB-modified microelectrode exhibited a sensitivity of $0.14 \pm 0.02 \mu \mathrm{A} / \mathrm{cm}^{2} \mathrm{nM}$ and an LOD value of $28.6 \pm 1.3 \mathrm{nM}$. The LOD value is well below the clinical threshold. This proof-of-concept study can be further miniaturized to point-of-care devices and integrated into wearable, handheld devices for better management of schizophrenia treatment.

\section{Materials and methods}

\section{Chemicals}

The following materials have been used without further purification: OLZ (catalog number: 1478301, Merck),

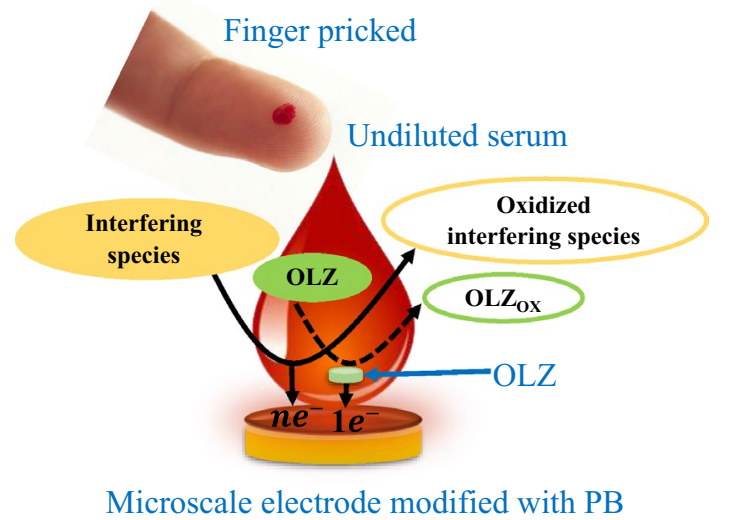

Fig. 1 Scheme of olanzapine detection in microliter volumes of undiluted serum samples using a platinum black-modified microelectrode 
Dihydrogen hexachloroplatinate (IV) hexahydrate (chloroplatinic acid; catalog number: 011051, Alfa aesar), 99\% Lead(II) acetate trihydrate (lead acetate; catalog number; A11746, Alfa Aesar), di-sodium hydrogen phosphate dihydrate (catalog number: 1.06580 .1000 , Merck), sodium dihydrogen phosphate dihydrate (catalog number: 1.06342.0250, Merck), sodium chloride (catalog number: 1.06404.1000, Merck), potassium hexacyanoferrate(II) trihydrate ('Ferrocyanide', catalog number: 1.04984.0100, Merck), potassium hexacyanoferrate(III) ('Ferricyanide', catalog number: 1.04973.0100, Merck), acetone Find Bio-Lab, Ltd.), 2-propanol (catalog number: 001626052100, Bio-Lab, Ltd.) and hydrochloric acid 32\% (catalog number: 000846050100, Bio-Lab, Ltd.). Ultra-pure water ( $>18 \mathrm{M} \Omega$ ) was obtained from a Super Q water system (Millipore). All prepared solutions were diluted in PBS $(10 \mathrm{mM}, \mathrm{pH}$ 7.4) solution. For all the electrochemical tests, a three-electrode electrochemical cell, consisting of an $\mathrm{Ag} / \mathrm{AgCl}$ reference electrode (a metal wire coated with $\mathrm{Ag} / \mathrm{AgCl}$ ink; catalogue number: 011464, BAS, Inc.), a ring platinum counter electrode (catalogue number: 012961, ALS Co., Ltd.), and microfabricated microelectrodes as working electrodes were used.

\section{Preparation of buffered samples}

A stock solution of $10 \mathrm{mg} / \mathrm{ml} \mathrm{OLZ} \mathrm{was} \mathrm{prepared} \mathrm{in} \mathrm{2-pro-}$ panol and stored at $-20^{\circ} \mathrm{C}$. OLZ standard solutions $(100 \mu \mathrm{l}$ each) of 20,50,90,120, and $150 \mathrm{nM}$ were prepared by diluting the $10 \mathrm{mg} / \mathrm{ml} \mathrm{OLZ} \mathrm{stock} \mathrm{solution} \mathrm{with} \mathrm{PBS} \mathrm{(10} \mathrm{mM,}$ pH 7.4).

\section{Preparation of undiluted serum samples}

Serum samples were collected from a healthy 38-year-old adult volunteer. To prepare the serum samples, blood samples were collected in $15 \mathrm{ml}$ tubes (BD Vacutainer® SSTTM II Advance, Fisher Scientific, Ltd.) and left for $30 \mathrm{~min}$ to allow the blood to clot. The clotted blood was centrifuged (1200 rpm for $10 \mathrm{~min}$ ), and the supernatant was collected and stored in a new $15 \mathrm{~mL}$ tube. Following another centrifugation step (1200 rpm for $10 \mathrm{~min}$ ), the supernatant was collected and $0.5 \mathrm{~mL}$ aliquots were stored in $1.5 \mathrm{~mL}$ Eppendorf tubes at $-20^{\circ} \mathrm{C}$. OLZ standard solutions (100 $\mu \mathrm{l} \mathrm{each)}$ of $20,50,90,120$, and $150 \mathrm{nM}$ were prepared by diluting the $10 \mathrm{mg} / \mathrm{ml} \mathrm{OLZ} \mathrm{stock} \mathrm{solution} \mathrm{with} \mathrm{an} \mathrm{undiluted} \mathrm{serum}$ sample. Ethical approval was obtained from the Ben-Gurion University of the Negev (BGU) Human Subjects Research Committee.

\section{Microfabrication of gold microelectrodes}

A borosilicate glass substrate was cleaned with acetone, isopropanol, and deionized (DI) water and then dried with nitrogen gas. The photoresist (AZ 5214E) coating process starts with spinning the wafer with the photoresist at 2200 RPM for $12 \mathrm{~s}$, followed by a soft bake on a contact hot plate at $110{ }^{\circ} \mathrm{C}$ for $2 \mathrm{~min}$ and $30 \mathrm{~s}$. Next, the electrode patterns were transferred from the mask using a hard contact of 7.6 $\mathrm{mW} / \mathrm{cm}^{2}$ for $65 \mathrm{~s}$ using a mask aligner (Karl Suss Mask Aligner MA6). The exposed wafer was then developed in AZ 726 MIF developer for $6 \mathrm{~min}$, followed by rinsing in DI water for $5 \mathrm{~min}$. Next, $20 \mathrm{~nm}$ of titanium and $200 \mathrm{~nm}$ of gold were deposited using the E-gun deposition system. The wafer was then transferred to a beaker with acetone solution for a lift off process that resulted in the Au/Ti microelectrode patterns on a glass substrate. The wafer was again rinsed in the DI water for $1 \mathrm{~min}$ to remove any residue from the wafer. SU8-3005 was used to define the microelectrode chamber; this allows cleaning the microelectrode with an AMI (acetone, methanol, and isopropanol) solution without destroying the chamber before using it. The process starts with spin coating SU8-3005 at 3000 RPM for $30 \mathrm{~s}$, followed by a soft bake on a contact hot plate at $95{ }^{\circ} \mathrm{C}$ for $15 \mathrm{~min}$. Next, the photoresist was exposed to light through the electrodes' mask using a hard contact of $7.6 \mathrm{~mW} / \mathrm{cm}^{2}$ for $50 \mathrm{~s}$ at a Mask Aligner (MA6, SUSS MicroTec). Post Exposer Bake (PEB) for $5 \mathrm{~min}$ at $95^{\circ} \mathrm{C}$ was used, since this is a negative photoresist and the wafer was cooled down to room temperature. The exposed wafer was then developed in PGMA ERB developer solution for $8 \mathrm{~min}$ and washed in isopropanol solution for $10 \mathrm{~s}$. The hard bake on a contact hot plate at $150{ }^{\circ} \mathrm{C}$ for $5 \mathrm{~min}$ was carried out to remove any hydration on the substrate, and oxygen plasma cleaning ( $30 \mathrm{~W}, 500 \mathrm{mTorr}, 2 \mathrm{~min}, 3 \mathrm{sccm}$ ) was used after the hard bake to remove any residues or impurities on the substrate. The wafer was then coated with photoresist before dicing it into chips using a Dicing saw (ADT-7100). All chips were cleaned with AMI solution before use. The microelectrode microfabrication flow process is shown in Fig. 2.

\section{Preparing platinum black deposition solution}

Platinum black deposition solution was prepared by mixing $1 \%$ chloroplatinic acid and $0.05 \%$ lead acetate in DI water. The mixture was then stirred and $0.0025 \%$ of $32 \%$ hydrochloric acid was added to the mixture (Stanca et al. 2017). The prepared solution was covered with aluminum foil and stored at room temperature.

\section{Platinum black electrodeposition onto microscale electrodes}

Before the gold microelectrode was modified, the chip was cleaned with AMI solution, followed by rinsing in DI water. The chip was then dried with nitrogen. The 
$\mathbf{A}$

(i)

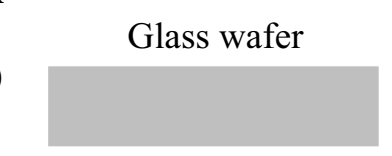

(ii)

Spin coating and exposure

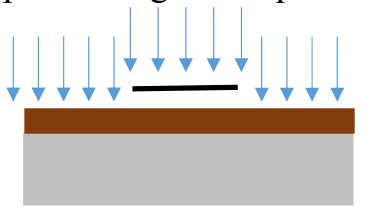

(iii)

(iv)

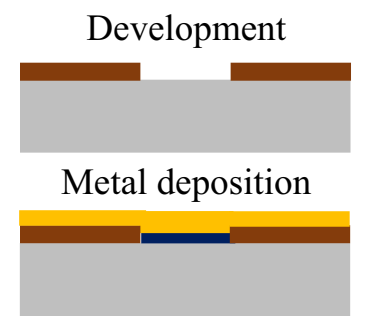

(v)

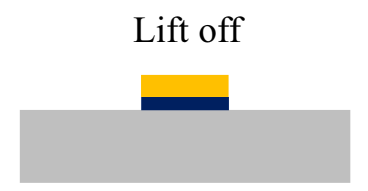

Spin coating and exposure

(vi)
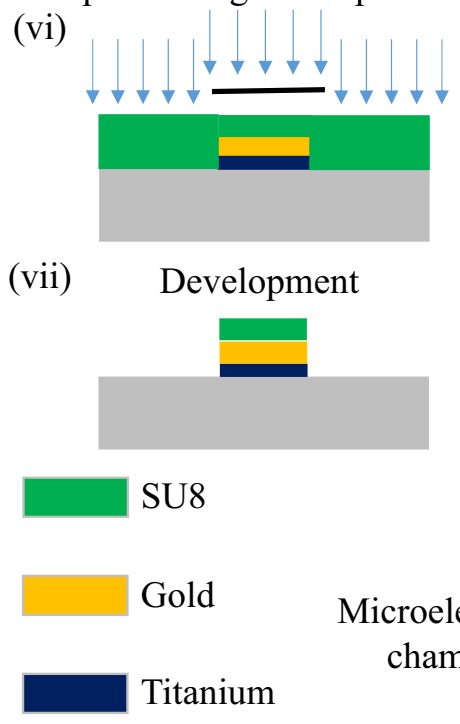

Photoresist

Glass wafer
B

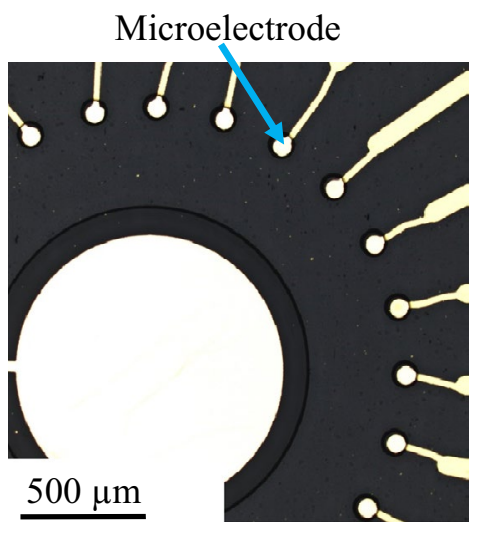

C

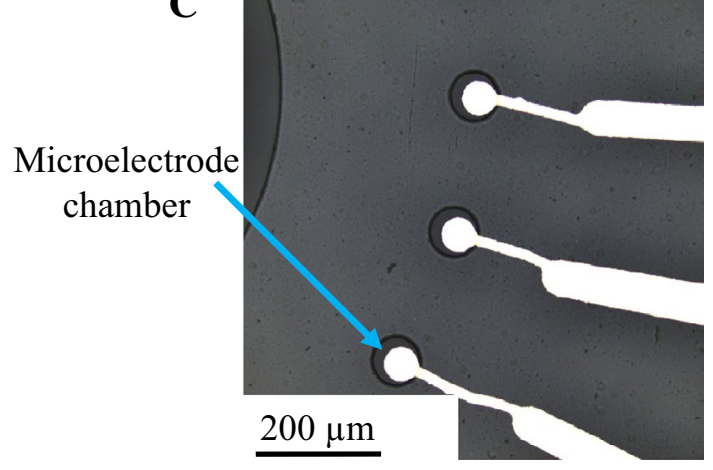

Fig. 2 a Microfabrication of gold microelectrodes: (i) a cleaning glass wafer, (ii) the spin coating of the photoresist followed by exposure using the mask aligner, (iii) a developing patterned wafer in 726MIF developer solution, (iv) Ti deposition followed by Au onto the patterned photoresist, (v) metal lift off in acetone solution resulting in the Ti/Au microelectrode patterns, (vi) the spin coating SU8

chronopotentiometry technique (current density $=4.8 \mathrm{~mA} /$ $\mathrm{cm}^{2}$, time $=5 \mathrm{~min}$ ) was used modify the gold microelectrode.

\section{Surface characterization}

The PB-modified microelectrode was visually inspected using an optical microscope; then the morphology of the PB surface was observed using a high-resolution scanning electron microscope (HRSEM; JSM-7400F-JEOL). The thickness of the PB film was characterized using Dektak profiler (Veeco Dektak-8).

\section{Electrochemical characterization}

PB-modified electrodes were electrochemically characterized in $10 \mathrm{mM}$ PBS (pH 7.4) and $5 \mathrm{mM}$ ferricyanide/ ferrocyanide solution. Cyclic voltammetry $\left(\mathrm{CV} ; E_{\mathrm{i}}=0 \mathrm{~V}\right.$, $E_{1}=-0.2 \mathrm{~V}, E_{2}=0.65 \mathrm{~V}$, scan rate $=0.1 \mathrm{~V} / \mathrm{s}$, number of scans $=2$ ) was used for testing the electrochemical activity. negative photoresist and exposure using the mask aligner for defining the microelectrode chamber, and (v) the development of the SU8 patterned wafer in PGMA developer to define the chamber. b A low-resolution optical image of the microelectrodes and $\mathbf{c}$ a high-resolution optical image of the microelectrodes showing the well-defined SU8 microchambers

\section{Olanzapine analysis in buffered and undiluted serum samples}

The electrochemical detection performance of OLZ was tested in both buffered and undiluted serum samples with a sensor array. The Differential Pulse Voltammetry technique (DPV; a potential sweep between -0.1 and $0.6 \mathrm{~V}$, a potential increment of $0.05 \mathrm{~V}$, an amplitude of $0.05 \mathrm{~V}$, a pulse width of $0.009 \mathrm{~s}$, a step height of $0.005 \mathrm{~V}$, and a step time of $0.1 \mathrm{~s}$ ) was used to obtain the dose response of OLZ; then the dose response plot was used to calculate the LOD and sensitivity. The slope of the linear regression of the dose response plot gives the sensitivity, whereas LOD is calculated as 3 standard deviations of the background signal. The repeatability of the PB-modified microelectrode was tested for detecting $150 \mathrm{nM} \mathrm{OLZ-spiked} \mathrm{undi-}$ luted serum samples. For this experiment, two microelectrodes were modified and the measurement was repeated 10 times for both microelectrodes. The PB-modified microelectrode was cleaned in between the measurements with PBS and DI water, followed by gently soaking the liquid with fine wipes. 


\section{Results and discussion}

\section{Platinum black-modified microelectrodes: modification and characterization}

\section{Microelectrode modification with platinum black and its electrochemical characterization}

In order to selectively electrodeposit PB on the Au microelectrodes, the working microelectrode portions were immersed in the electrodeposition solution and connected accordingly. A constant cathodic current density of $4.8 \mathrm{~mA} /$ $\mathrm{cm}^{2}$ was applied to the working microelectrode for $5 \mathrm{~min}$ against a ring platinum counter electrode. The PB-modified $\mathrm{Au}$ microelectrode was then cleaned with DI water and dried with nitrogen. Figure 3 a shows the potential versus the time characteristics during the electrodeposition. The electrochemical response of the modified microelectrode was tested in $10 \mathrm{mM}$ PBS solution, followed by $5 \mathrm{mM}$ ferrocyanide/ferricyanide solution. Figure $3 b$ shows the CVs of the bare and the PB-modified microelectrode in buffered solution; this indicates the background signal (capacitive current). The PB-modified microelectrode exhibits a higher capacitive current because of its higher surface area, compared with the bare Au microelectrode. Figure $3 \mathrm{c}$ shows the $\mathrm{CVs}$ of the bare and PB-modified microelectrode in $5 \mathrm{mM}$ ferrocyanide/ferricyanide. The electrochemical response in a $5 \mathrm{mM}$ ferrocyanide/ferricyanide redox couple indicates that there was a higher oxidation current for the PB-modified microelectrode, compared with the bare one due to its high effective surface area.

We also calculated the effective surface area of the PBmodified microelectrodes using $\mathrm{CV}$ at different scan rates of $0.5,0.4,0.3,0.2,0.1$, and $0.05 \mathrm{~V} / \mathrm{s}$ in a $5 \mathrm{mM}$ ferrocyanide/ ferricyanide solution. The potential was scanned from -0.2 to $0.65 \mathrm{~V}$ for 2 cycles. The oxidation current peak for the PB-modified microelectrode was plotted against the square root of the scan rate, and the effective surface area of the modified microelectrode was calculated (Fig. 3e).

We calculated the effective surface area of the modified electrode by using the Randles-Sevcik relationship in Eq. 1 (Bard and Faulkner 2001):
A

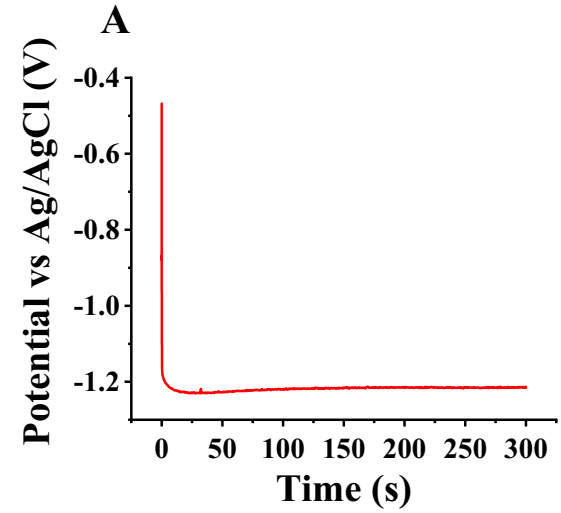

D

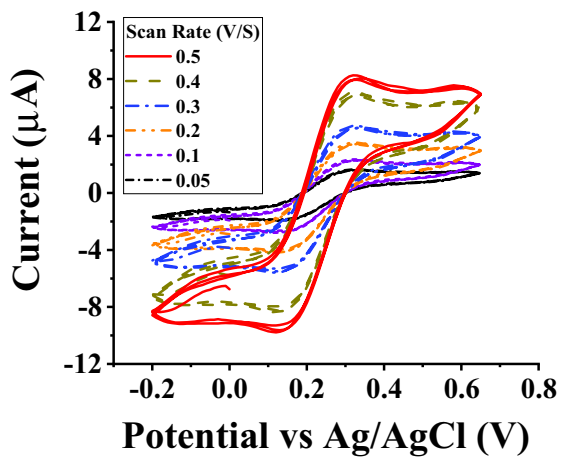

B

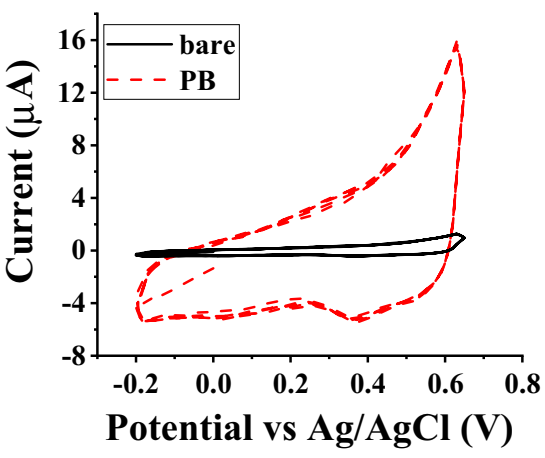

$\mathbf{E}$

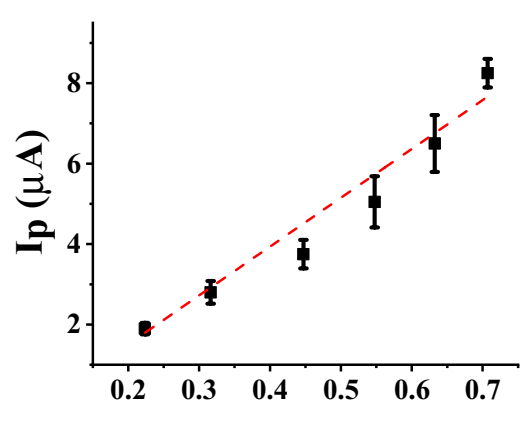

(Scan rate $)^{0.5}(\mathrm{~V} / \mathrm{s})^{0.5}$
C

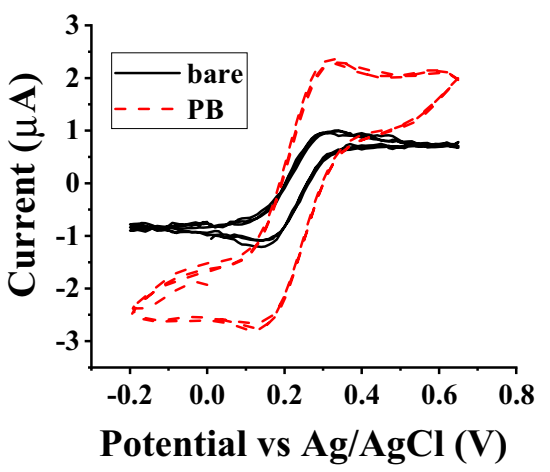

$\mathbf{F}$

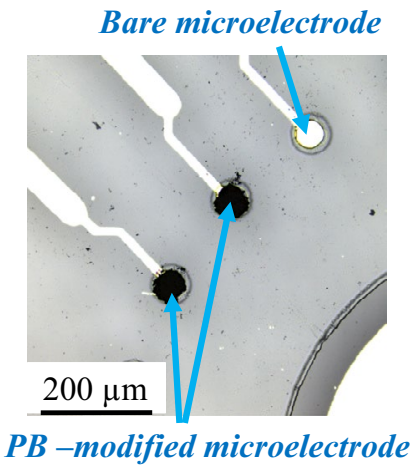

Fig. 3 a Electrodeposition of $\mathrm{PB}$ on a gold microelectrode using Chronopotentiometry, b CVs measured in $10 \mathrm{mM}$ PBS solution, and c in $5 \mathrm{mM}$ ferrocyanide/ferricyanide at a scan rate of $0.1 \mathrm{~V} / \mathrm{s}$ using a bare (black solid) and a PB-modified microelectrode (red dashes), d CVs measured at scan rates of 0.5 (solid red), 0.4 (dark yellow dashes), 0.3 (blue dashed dots), 0.2 (orange dashed dots), 0.1 (violet short dashes), and 0.05 (short black dashed dots) V/s by using a PBmodified microelectrode, $\mathbf{e}$ peak current vs the square root of the scan rate, and $\mathbf{f}$ an optical image of the PB-modified Au microelectrode 
$I_{\mathrm{p}}=0.4463\left(F^{3} / R T\right)^{1 / 2} n^{3 / 2} A_{\mathrm{eff}} D^{1 / 2} C^{*} v^{1 / 2}$,

where $I_{\mathrm{p}}[\mathrm{A}]$ is the peak current, $F[\mathrm{C} / \mathrm{mol}]$ is the Faraday constant, $R[\mathrm{~J} / \mathrm{mol} \mathrm{K}]$ is the universal gas constant, $T[\mathrm{~K}]$ is the absolute temperature, $n$ is the number of moles of electrons transferred in the cell reaction $(n=1$ for a ferrocyanide/ ferricyanide redox reaction), $A_{\text {eff }}\left[\mathrm{cm}^{2}\right]$ is the active surface area of the electrode, $D\left[\mathrm{~cm}^{2} / \mathrm{s}\right]$ is the diffusion coefficient of the electro-active species $\left[0.72 \times 10^{-5} \mathrm{~cm}^{2} / \mathrm{s}\right.$ for ferricyanide and $0.67 \times 10^{-5} \mathrm{~cm}^{2} / \mathrm{s}$ for ferrocyanide (Konopka and McDuffie 1970)], $C^{*}\left[\mathrm{~mol} / \mathrm{cm}^{3}\right]$ is the bulk concentration of the electro-active species, and $v[\mathrm{~V} / \mathrm{s}]$ is the linear potential scan rate.

The calculated $A_{\text {eff }}$ value of the PB-modified microelectrode was found to be $3.5 \times 10^{-3} \mathrm{~cm}^{2} \pm 0.1 \times 10^{-3} \mathrm{~cm}^{2}$, which is 4.5 times higher than that of the bare Au microelectrode (the $\underline{A}_{\text {eff }}$ value of the Au microelectrode calculated from the mask design is $7.8 \times 10^{-4} \pm 0.2 \times 10^{-4} \mathrm{~cm}^{2}$ ). The higher surface area of the PB-modified microelectrode is due to the non-homogenous nature and the roughness of the PB material and is equivalent to 3 layers of PB (Wayu et al. 2016). Lastly, the optical image of the PB-modified microelectrode was recorded for visualizing the modification. Figure $3 \mathrm{f}$ shows the PB-modified and bare Au microelectrodes. We noted that despite the assumption of linearity in Fig. 3e, the curve exhibited some non-linearity. The non-linearity can be due to the different conditions used to derive Eq. (1) and the experimental data. For example, Eq. (1) was calculated, based on the assumption of semi-infinite linear diffusion. However, we recorded electrochemical signals from a sample drop that can contribute to a non-linear restricted diffusion element due to the limited diffusion attributed to the drop's volume. Moreover, Eq. (1) was calculated for macroscale electrodes under equilibrium conditions, whereas we used microelectrodes that increase the impact of the kinetic parameters (the electron transfer rate).

\section{Surface characterization}

The thickness of the PB-modified microelectrode was measured using the Dektak profiler, which showed a thickness of $6.5 \mu \mathrm{m}$ (Fig. 4a). The PB-modified microelectrode was characterized using HRSEM to visualize the presence of PB on the Au microelectrodes. Figure 4b, c shows the lowand high-resolution micrographs of the bare gold microelectrode; Fig. 4d, e shows the surface of the PB-modified microelectrode, which is indicative of the presence of $\mathrm{PB}$ on the Au microelectrodes.

\section{Olanzapine sensing performance using a platinum black-modified microelectrode}

Olanzapine sensing performance for the bare and the PBmodified microelectrode was tested in $20 \mu \mathrm{l}$ of buffered samples. Differential pulse voltammetry was used to record
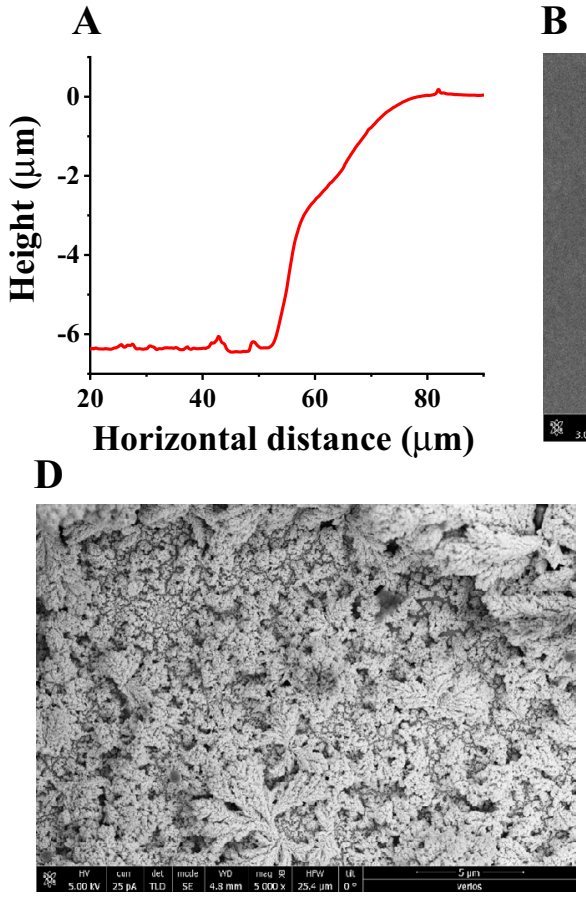

\section{B}

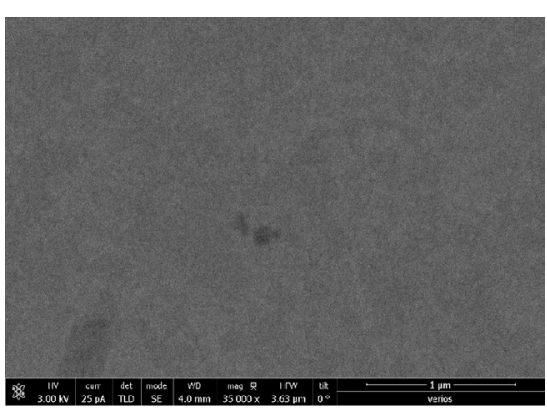

E

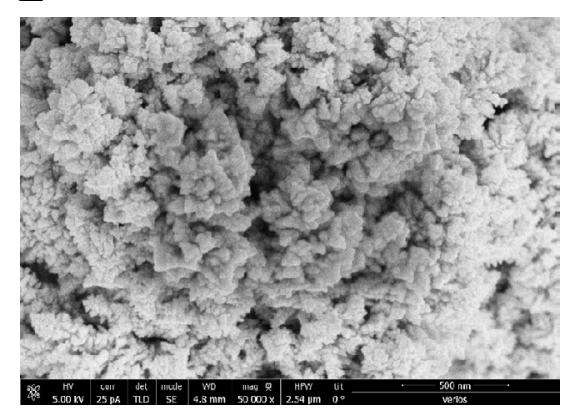

C

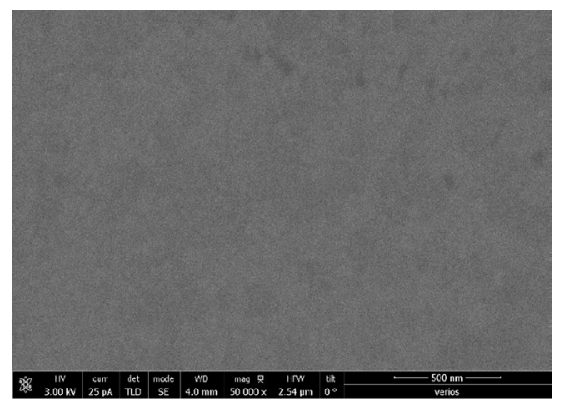

Fig. 4 a Thickness of the PB film using the Stylus profiler, a low and high magnification micrograph of the bare $\mathbf{b}$ low, and $\mathbf{c}$ high magnification, and a PB-modified microelectrode $\mathbf{d}$ low, and e high magnification 
the OLZ oxidation for different concentrations. A positive dose response was observed for the bare (Fig. 5a) and the PB-modified microelectrodes (Fig. 5b). The linear regression analysis of the positive dose response plot was used to calculate the sensitivity and the LOD (Eqs. 2 and 3).

Bare microelectrode $\left(R^{2}=0.97 ; \mathrm{LOD}=80.0 \pm 13.3 \mathrm{nM}\right)$ :

$$
\begin{aligned}
j_{\text {anodic peak }}= & \left(0.030 \pm 0.005 \mu \mathrm{A} / \mathrm{cm}^{2} \mathrm{nM}\right)[\mathrm{OLZ}] \\
& +\left(1.6 \pm 0.8 \mu \mathrm{A} / \mathrm{cm}^{2}\right)
\end{aligned}
$$

$P B-m o d i f i e d$ microelectrode $\quad\left(R^{2}=0.98 ;\right.$ $\mathrm{LOD}=15.6 \pm 0.8 \mathrm{nM})$ :

$$
\begin{aligned}
j_{\text {anodic peak }}= & \left(0.20 \pm 0.01 \mu \mathrm{A} / \mathrm{cm}^{2} \mathrm{nM}\right)[\mathrm{OLZ}] \\
& +\left(3.80 \pm 1.04 \mu \mathrm{A} / \mathrm{cm}^{2}\right)
\end{aligned}
$$

The dose response plot resulted in a sensitivity of $0.030 \pm 0.005 \mu \mathrm{A} / \mathrm{cm}^{2} \mathrm{nM}$ and $0.20 \pm 0.01 \mu \mathrm{A} / \mathrm{cm}^{2} \mathrm{nM}$, as well as an LOD value of $80.0 \pm 13.3 \mathrm{nM}$ and $15.6 \pm 0.8 \mathrm{nM}$ for the bare and PB-modified microelectrodes, respectively. The PB-modified microelectrode is 6.7 times more sensitive than the bare one and has 5.1 times better sensing performance in terms of LOD, compared with the bare microelectrode. The improved sensing performance is due to the high surface area of the PB-modified microelectrode.

\section{Olanzapine detection performance in undiluted serum using a platinum black-modified microelectrode}

The OLZ sensing performance was evaluated in a $20 \mu \mathrm{l}$ volume of an undiluted serum sample from a healthy volunteer using the bare and the PB-modified microelectrode. The DPVs were recorded for different concentrations of OLZ-spiked undiluted serum samples. The bare $\mathrm{Au}$ microelectrode was unable to differentiate between different OLZ concentrations (Fig. 6a), whereas the PBmodified microelectrode showed a positive dose response with a high background. The linear dose response plot of the PB-modified microelectrode resulted in a sensitivity of $0.14 \pm 0.02 \mu \mathrm{A} / \mathrm{cm}^{2} \mathrm{nM}$ and an LOD value of $28.6 \pm 1.3 \mathrm{nM}$. The LOD value is well below the clinical threshold.

$P B$-modified microelectrode $\quad\left(R^{2}=0.98 ;\right.$ $\mathrm{LOD}=27.8 \pm 3.9 \mathrm{nM})$ :
Fig. 5 OLZ detection in PBS samples. DPVs of 150 (solid red), 120 (dark yellow dashes), 100 (blue dashed dots), 80 (orange dashed dots), 50 (blue short dashes), and PBS (short black dashed dots) nM OLZ concentrations using a a bare and $\mathbf{b}$ a PB-modified microelectrode. c Dose response plot for bare (dark yellow rectangles) and a PB-modified microelectrode (red circles)
Phosphate-buffered saline
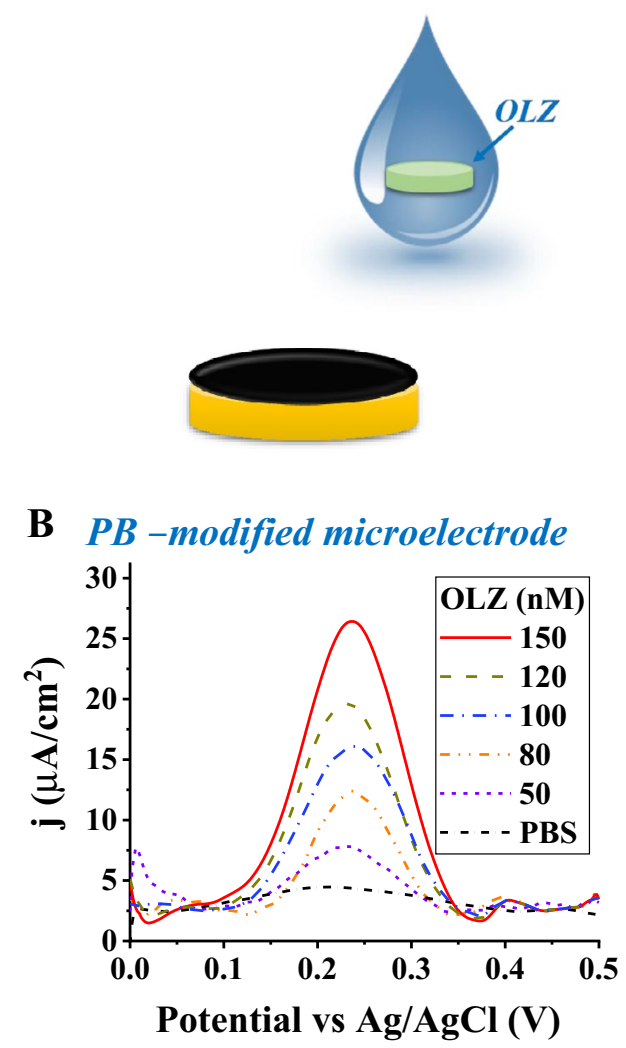

A Bare microelectrode

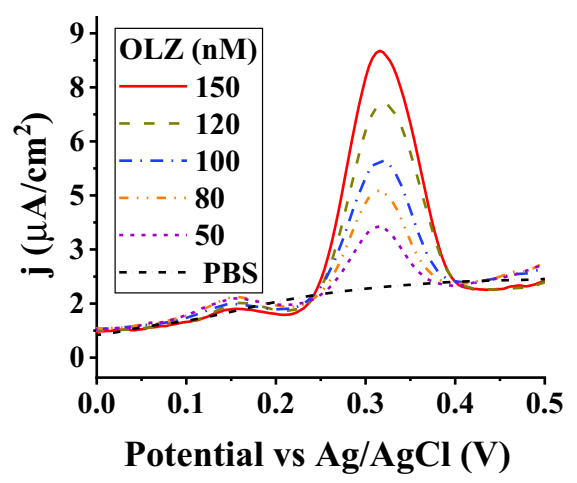

C

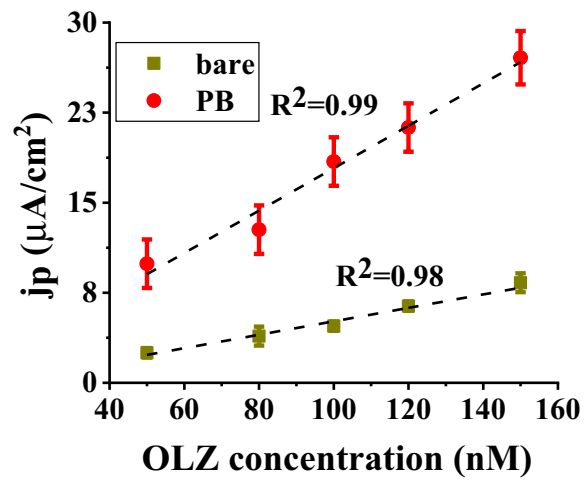




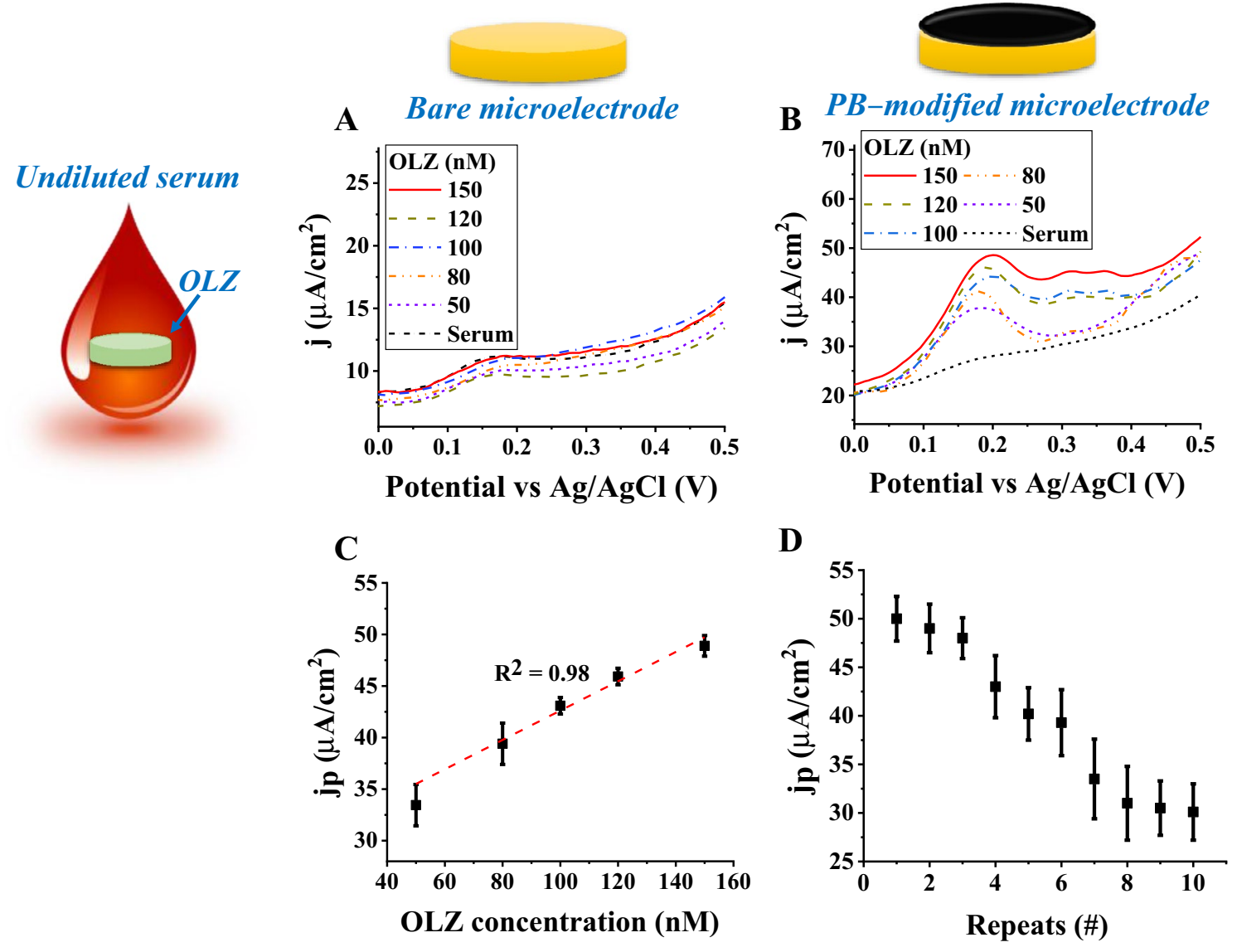

Fig. 6 OLZ detection in undiluted serum samples. DPVs of 150 (solid red), 120 (dark yellow dashes), 100 (blue dashed dots), 80 (orange dashed dots), 50 (blue short dashes), and PBS (black short dashed dots) nM OLZ concentrations using a a bare and b PB-modi-

$j_{\text {anodic peak }}=\left(0.14 \pm 0.02 \mu \mathrm{A} / \mathrm{cm}^{2} \mathrm{nM}\right)[\mathrm{OLZ}]+\left(28.6 \pm 1.3 \mu \mathrm{A} / \mathrm{cm}^{2}\right)$

The repeatability of the PB-modified microelectrode was tested for $150 \mathrm{nM}$ OLZ-spiked $20 \mu$ undiluted serum samples. In order to reduce the effect of fouling or residuals from the previously measured sample, the modified microelectrode was cleaned between consecutive measurements with PBS solution, followed by DI water cleaning, and then gently dried with fine wipes. At the 5th repetition, the PB-modified microelectrode was able to retain $80.6 \%$, and at the 10 th repetition, it was able to retain $60 \%$ of the current from the first measurement. fied microelectrodes. c Dose response plot for a PB-modified microelectrode (rectangular black). d Repeatability of the PB-modified microelectrode for OLZ sensing

\section{Conclusions}

Point-of-care monitoring of antipsychotics using electrochemical sensors shows great potential for the therapeutic management and treatment of schizophrenia. Modification of microelectrodes with PB enabled OLZ detection in an undiluted finger-pricked serum sample. By further miniaturizing and integrating PB-modified microelectrodes into point-ofcare testing devices, the management of schizophrenia treatment can be greatly improved. Our long-term goal is to build the capabilities needed to access complementary chemical information (e.g., from blood). OLZ detection from serum is a critical step in developing sensors for clinical analyses. 
Importantly, this sensor measurement requires minimal sample pretreatment (only centrifugation to generate the serum), which is in contrast to other approaches that employ additional steps to remove protein, dilute interferents, or adjust $\mathrm{pH}$ to facilitate analysis.

Acknowledgements We gratefully acknowledge financial support from the Brain and Behavior Research Foundation NARSAD Young Investigator Grant and the Jeanne Marie Lee Investigator Grant (Grant 26038) for funding this project. This research was also supported in part by the Helmsley Charitable Trust through the Agricultural, Biological and Cognitive Robotics Initiative and by the Marcus Endowment Fund both at Ben-Gurion University of the Negev. The authors also wish to thank the Krietman School for the Mid-way Negev fellowship for their support. Lastly, the authors thank Yacov Bernstein for expert assistance with the thermal evaporator and the E-gun, Alexander Kozlovsky for assistance with the mask aligner, Rotem Manor for assistance with the stylus profiler, Nadav Dharan and Abraham Reiner for assistance with the dicing saw, and Roxana Golan for expert assistance with the SEM system.

Open Access This article is licensed under a Creative Commons Attribution 4.0 International License, which permits use, sharing, adaptation, distribution and reproduction in any medium or format, as long as you give appropriate credit to the original author(s) and the source, provide a link to the Creative Commons licence, and indicate if changes were made. The images or other third party material in this article are included in the article's Creative Commons licence, unless indicated otherwise in a credit line to the material. If material is not included in the article's Creative Commons licence and your intended use is not permitted by statutory regulation or exceeds the permitted use, you will need to obtain permission directly from the copyright holder. To view a copy of this licence, visit http://creativecommons.org/licenses/by/4.0/.

\section{References}

Ahmed HM, Mohamed MA, Salem WM (2015) New voltammetric analysis of olanzapine in tablets and human urine samples using a modified carbon paste sensor electrode incorporating gold nanoparticles and glutamine in a micellar medium. Anal Methods-Uk 7:581-589. https://doi.org/10.1039/c4ay02450h

Arvand M, Palizkar B (2013) Development of a modified electrode with amine-functionalized $\mathrm{TiO} 2 /$ multi-walled carbon nanotubes nanocomposite for electrochemical sensing of the atypical neuroleptic drug olanzapine. Mater Sci Eng C Mater Biol Appl 33:4876-4883. https://doi.org/10.1016/j.msec.2013.08.002

Arvand M, Orangpour S, Ghodsi N (2015) Differential pulse stripping voltammetric determination of the antipsychotic medication olanzapine at a magnetic nano-composite with a core/shell structure. Rsc Adv 5:46095-46103. https://doi.org/10.1039/c5ra00061k

Bard AJ, Faulkner LR (2001) Electrochemical methods: fundamentals and applications, 2nd edn. Wiley

El-Shal MA (2013) Electrochemical studies for the determination of quetiapine fumarate and olanzapine antipsychotic drugs. Adv Pharm Bull 3:339-344. https://doi.org/10.5681/apb.2013.055
Konopka SJ, McDuffie B (1970) Diffusion coefficients of ferri- and ferrocyanide ions in aqueous media, using twin-electrode thinlayer electrochemistry. Anal Chem 42:1741-1746. https://doi. org/10.1021/ac50160a042

Li Y, Sella C, Lemaitre F, Collignon MG, Thouin L, Amatore C (2013) Highly sensitive platinum-black coated platinum electrodes for electrochemical detection of hydrogen peroxide and nitrite in microchannel. Electroanalysis 25:895-902. https://doi. org/10.1002/elan.201200456

Lin CH, Wang FC, Lin SC, Huang YH, Chen CC (2017) A randomized, double-blind, comparison of the efficacy and safety of low-dose olanzapine plus low-dose trifluoperazine versus full-dose olanzapine in the acute treatment of schizophrenia. Schizophr Res 185:80-87. https://doi.org/10.1016/j.schres.2017.01.004

Lu ML, Chen CH, Kuo PT, Lin CH, Wu TH (2018) Application of plasma levels of olanzapine and N-desmethyl-olanzapine to monitor metabolic parameters in patients with schizophrenia. Schizophr Res 193:139-145. https://doi.org/10.1016/j.schres.2017.07.022

Merli D, Dondi D, Pesavento M, Profumo A (2012) Electrochemistry of olanzapine and risperidone at carbon nanotubes modified gold electrode through classical and DFT approaches. J Electroanal Chem 683:103-111. https://doi.org/10.1016/j.jelec hem.2012.08.011

Ni XJ, Wang ZZ, Shang DW, Lu HY, Zhang M, Wen YG (2018) Simultaneous analysis of olanzapine, fluoxetine, and norfluoxetine in human plasma using liquid chromatography-mass spectrometry and its application to a pharmacokinetic study. J Chromatogr B Anal Technol Biomed Life Sci 1092:506-514. https://doi. org/10.1016/j.jchromb.2018.05.026

Rouhani M, Soleymanpour A (2019) A new selective carbon paste electrode for potentiometric analysis of olanzapine. Measurement 140:472-478. https://doi.org/10.1016/j.measurement.2019.04.018

Seo HK, Park DJ, Park JY (2008) Fabrication and characterization of platinum black and mesoporous platinum electrodes for in-vivo and continuously monitoring electrochemical sensor applications. Thin Solid Films 516:5227-5230. https://doi.org/10.1016/j. tsf.2007.07.098

Stanca SE, Hanschke F, Ihring A, Zieger G, Dellith J, Kessler E, Meyer HG (2017) Chemical and electrochemical synthesis of platinum black. Sci Rep 7:1074. https://doi.org/10.1038/s41598-017-01040 $-8$

Wayu MB, Pannell MJ, Leopold MC (2016) Layered xerogel films incorporating monolayer-protected cluster networks on platinumblack-modified electrodes for enhanced sensitivity in first-generation uric acid biosensing. ChemElectroChem 3:1245-1252. https ://doi.org/10.1002/celc.201600164

Wu TH, Chiu CC, Chen PY, Huang MC, Chen CH, Shen WW, Lu ML (2019) Obsessive-compulsive symptoms in patients with schizophrenia: Relationships with olanzapine pharmacological parameters, psychopathology, and quality of life. Psychiatry Res 276:1-5. https://doi.org/10.1016/j.psychres.2019.03.007

Publisher's Note Springer Nature remains neutral with regard to jurisdictional claims in published maps and institutional affiliations. 